\title{
La cátedra integradora en las mallas curriculares de las carreras universitarias
}

\author{
María Adela Valdés Sáenz ${ }^{1}$ \\ Yoel Rodríguez Guerra² \\ Katia Díaz Valdés ${ }^{3}$
}

\section{RESUMEN}

Uno de los propósitos de la Educación Superior en Latinoamérica es la formación integral del individuo, lo que significa cultivar todos los aspectos de la personalidad humana: física, intelectual y moral, aunque esta propuesta en este nivel educacional no es nueva, lo cierto es que actualmente existe consenso en cuanto a la necesidad de recuperar esta finalidad durante tanto tiempo ignorada. En aras de lograr lo que se demanda actualmente en la aplicación de los rediseños curriculares por parte de los actores pertenecientes a las instituciones universitarias se perfila la figura de la cátedra integradora que en los semestres académicos, supone identificar los objetivos principales a lograr en ese nivel, para lo cual es necesario evaluar, con un enfoque integrador, la contribución de cada una de las materias que intervienen, de modo tal, que el resultado caracterice como sistema, las nuevas cualidades que han de revelarse en el estudiante como resultado de su proceso de formación, además de que ofrece una visión del momento del proceso de formación y actúa como sistema para lograr determinados objetivos, tanto de tipo instructivo como educativo, que rebasan el alcance de cada una de las asignaturas en particular. Este trabajo pretende demostrar las actividades sistémicas que requiere la coordinación de la cátedra integradora dentro del semestre académico para que cumpla a plenitud con la función didáctica que le corresponde y para la cual se estableció en los rediseños de carreras universitarias.

Palabras clave: cátedra integradora, integración horizontal, diseño de mallas curriculares, procesos de formación, educación superior.

Recibido: 31 de mayo de 2017

Aceptado: 14 de noviembre de 2017

1 Doctora en Ciencias Forestales. Magister en Agroecología y Agricultura Sostenible. Profesora e investigadora en la Universidad Estatal Amazónica. Con experiencia laboral en la docencia universitaria. Autora de artículos. Participación en Congresos y Eventos locales, nacionales e internacionales.Correo electrónico: madelavaldes@gmail.com

2 Doctor en Ciencias Forestales. Magister en Ecología. Director de la Oferta Académica El Pangui, profesor e investigador en la Universidad Estatal Amazónica. Autor de artículos en Etnobotánica, Agroecología y Silvicultura. Participación en Congresos y Eventos locales, nacionales e internacionales.

3 Doctora en Estomatología. Especialista de I grado en Estomatología General Integral. Magister en Salud Bucal Comunitaria. Profesora principal de Materiales Dentales, Radiología y Prótesis Estomatológicas. 


\section{The integrating professorship in the curricular program of majors at universities}

\section{RESUMEN}

One of the purposes of Higher Education in Latin America is the integral formation of the individual, which means cultivating all aspects of the human personality: physical, intellectual and moral, although this proposal at this educational level is not new. Currenly, there is a consensus on the need to retrieve this purpose that has been ignored for a long time. In order to achieve what is being demanded in the application of the curricular redesigns by the actors universy actors to figure the integrating professorship, the academic semesters implies identifying the main objectives to be achieved at that level, for which it is necessary to evaluate, with an integrative approach, the contribution of each of the subjects involved, so that, the result characterizes, as a system, the new qualities that are to be revealed in the student as a result of process of training process, also, it provides a vision of the training process and acts as a system to achieve certain objectives, instructional and educational, that go beyond the scope of each particular subject. This written assignment demonstrates the systemic activities required by the coordination of the professorship integrating within the academic semester so that it fulfills the didactic function that corresponds for connection and established in the redesigns of major at universities.

Keywords: integrative chai, horizontal integration, design of curricula mesh, training processes, higher education.

\section{INTRODUCCIÓN}

Actualmente se habla de una nueva universidad, con un sistema de enseñanza aprendizaje nuevo, innovador y al mismo tiempo, de calidad, con estilos de aprendizaje centrados en la formación integral del estudiante, donde el profesor universitario además de impartir clases, debe fomentar que el estudiante reflexione por sí mismo, (García y de la Cruz, 2014).

El desarrollo de la educación superior en América Latina ha seguido, históricamente, derroteros similares a los que se han manifestado en el conjunto de la educación superior de otros continentes. No obstante, en su última etapa, ha venido enfrentando un conjunto de cambios que son parte de las transformaciones sociales más generales que vive la región. Estos cambios abarcan gradualmente al conjunto de funciones universitarias y en particular a la actividad de investigación e innovación, la cual se adentra en la compleja tarea de hacer del conocimiento una herramienta de transformación social. Lo anterior ha implicado ajustes al conjunto de funciones universitarias 
que van desde mejores definiciones al currículo de las distintas carreras para que cumpla funciones de desarrollo investigativo, hasta el fortalecimiento de las bases de desarrollo de las ciencias naturales y técnicas para que puedan cumplir con el objetivo de lograr resultados en campos del conocimiento históricamente no atendidos. Plantean además García y de la Cruz (2014), que estos recursos de aprendizaje se corresponden con los objetivos, los métodos y el nivel de comprensión de los educandos y están vinculados con lo que se espera que el estudiante aprenda, se cumple así con un algoritmo que favorece la asimilación de los contenidos. Se trata de que los estudiantes adquieran conocimientos y desarrollen habilidades, capacidades, actitudes y valores que les permitan responder de forma efectiva y oportuna a los diversos problemas presentes y futuros de una sociedad cada vez más compleja y dinámica

Las estrategias curriculares de una carrera constituyen una forma particular de desarrollar el proceso de enseñanza-aprendizaje con una direccionalidad altamente coordinada que responda al perfil de salida de la profesión en la que se imbrican de manera creciente los contenidos y los diversos métodos teóricos y prácticos de las unidades curriculares del plan de estudio que intervengan en ella.

La educación academicista centrada en planteamientos eruditos que no responden a las necesidades culturales e intelectuales contemporáneas, corre el riesgo de formar ciudadanos con carencias formativas, lo cual dificultará su desenvolvimiento en el mundo que les toque vivir, ello es aplicable a la enseñanza superior y en este sentido, una buena estrategia pedagógica será la que trate de lograr una acción formativa integral, que incluya de forma equilibrada, tanto los aspectos intelectuales como los valores, que potencian el desarrollo armónico del estudiante, sin olvidar la práctica y el contexto social en que se desarrollan, (Sierra, et.al.,2009).

Por supuesto, ello requiere del rompimiento de barreras, lo cual implica concebir el conocimiento como un todo donde se integren los elementos esenciales en contraste con el afán de alcanzar niveles cada vez más exactos en el conocimiento específico de una parte de la realidad. Una de las dificultades a considerar con especial atención es que la mayoría de los profesores que hoy están en los claustros universitarios no se han formado en escuelas pedagógicas, sino que por una u otra razón, han asumido la tarea de enseñar. La escasa atención brindada a la capacitación de los docentes, su selección, preparación o permanencia, sin modificar sustancialmente las actitudes, habilidades y capacidades profesionales de los maestros, sin un ejercicio previo de análisis y reflexión, sin los necesarios mecanismos y estímulos que permitan al profesor incorporarse al cambio, acarrean reformas curriculares que en la práctica quedan como un aparente hacer, sin mayor trascendencia. Si el profesor, quien va a dar vida y poner en práctica el nuevo currículo no lo comprende, no está convencido de él y no es tomado en cuenta en los procesos previos, el resultado es que no lo hace suyo y la posibilidad de cambio resulta casi nula. 
El diseño de una carrera constituye una forma particular de desarrollar el proceso de enseñanzaaprendizaje con una direccionalidad altamente coordinada que responda al perfil de salida de la profesión en la que se imbrican, de manera creciente, los contenidos y los diversos métodos teóricos y prácticos de las unidades curriculares de la malla curricular que intervengan en ella. Una buena estrategia pedagógica será la que trate de lograr una acción formativa integral, que incluya de forma equilibrada, tanto los aspectos intelectuales como los valores, que potencian el desarrollo armónico del estudiante, sin olvidar la práctica y el contexto social en que se desarrollan. Sierra, et.al.,(2009), indica que lo expresado anteriormente tendrá una influencia valiosa en el proceso formativo solo si se toman en cuenta desde el diseño de la malla curricular hasta el trabajo cotidiano en el aula o en las actividades prácticas que se realicen en los escenarios docentes reales, todo lo cual será posible en la medida en que en los diferentes centros se organicen las estructuras académicas funcionales que dirijan el desarrollo de dichas estrategias en el proceso formativo y la cátedra rectora o coordinadora de cada estrategia tenga el poder de convocatoria de las restantes asignaturas participantes de manera que se logre una adecuada coordinación y potenciación de las acciones en los aspectos que corresponda. Cualquiera que sea la organización que se les dé en uno u otro plan de estudio, las estrategias curriculares que se diseñen deberán posibilitar en su concepción y organización, una ruta de acción, una metodología, el deber ser y la integración de las propuestas así como las acciones curriculares correspondientes.

Perafán (2004) y Aldana y Joya (2008), coinciden en que se requiere propiciar espacios de reflexión orientados a la identificación y cambio de las concepciones epistemológicas de los docentes, de modo que se evidencien las diferentes formas de asumir el rol de docente y se centre la atención no solo en transmitir conocimientos, sino en aportar a la formación del estudiante, por lo menos, en tres aspectos: conceptos significativamente comprendidos e integrados a su cotidianidad, habilidades para resolver problemas de manera crítica y creativa y actitudes positivas hacia el conocimiento y la investigación En el desarrollo de una reforma curricular, así como en el desarrollo del currículo todos los involucrados aprenden, en especial los docentes deben ser vistos como sujetos que aprenden, que van construyendo su propio conocimiento sobre la docencia, de la misma manera en que los alumnos lo hacen, si pensamos que los únicos sujetos de aprendizaje son los alumnos, estamos negando las posibilidades de un crecimiento permanente, comunitario y colaborativo para todos los miembros de la institución tal y como se declara que se quiere promover para los alumnos.

Otra de las consecuencias de la dispersión de los contenidos en el currículo es que la motivación hacia la especialidad no alcanza los niveles deseados, fundamentado en un conocimiento parcial e insuficiente del contenido de la futura profesión y problemas en cuanto al estudio relacionados con la planificación del tiempo para el estudio, las técnicas de lectura y anotación, las costumbres de estudioy la concentración, tal y como señalan Cuza y Guibo (2015) y Valdés, Valdés y Fernández Oliva (2016).

La propuesta presentada en este trabajo está enfocada al perfeccionamiento de la cátedra integradora en la aplicación de los rediseños curriculares por parte de los actores pertenecientes 
a las instituciones universitarias. Todo depende, en gran medida, del esfuerzo que se le dedique al correcto desarrollo de este proceso y del interés por solucionar las dificultades que puedan ser encontradas en cada una de sus etapas.

\section{DESARROLLO}

Uno de los fines de la educación es la formación de seres humanos acorde a las exigencias de la globalización. Avilés (2011), plantea que parece ser urgente "transformar paradigmas educativos en función de garantizar aprendizajes de calidad tendientes al desarrollo humano para todos a lo largo de la vida. Este propósito, encuentra sustento teórico en los llamados Pilares básicos de la Educación del Siglo XXI, definidos por la UNESCO: aprender a conocer, aprender a ser, aprender a hacer, aprender a vivir juntos y aprender a emprender.

El enfoque sistémico de las carreras universitarias resulta conveniente destacarlo desde uno de sus rasgos fundamentales: un sistema es tal, cuando la integración de sus partes trae consigo una nueva cualidad, que no estaba presente en ninguna de esas partes. Según Silva (2006), esa idea deviene en hilo conductor de estos razonamientos relacionados con el enfoque de la carrera como sistema. Así, en el enfoque en sistema de la carrera, es posible identificar subsistemas de orden menor, cada uno de los cuales tiene una cualidad nueva que lo caracteriza como tal. Davidov (1987), señala que la asimilación del conocimiento comprende la estructuración previa del objeto de estudio y la formación de capacidades para establecer las relaciones teóricas entre las cosas.

Si bien la carrera, de acuerdo con ese esquema, es el resultado de la integración de las diferentes asignaturas, resulta difícil lograr que ellas se integren al logro de los objetivos más generales; lo que casi siempre ocurre es que cada una actúa con cierta independencia de las restantes, sin un análisis pedagógico profundo acerca de si sus efectos convergen hacia los objetivos deseados en la formación de dicho profesional. Resulta necesario reflexionar sobre este problema, teniendo en cuenta que en una carrera pueden impartirse entre 54 y 72 como promedio. ¿Funcionará realmente como sistema una carrera en la que cada una de ellas actúe de forma independiente, sin una adecuada coordinación de las acciones a realizar en aras de objetivos comunes? La respuesta es evidente.

Una visión integradora del semestre debe ser capaz de precisar sus propósitos esenciales, unos pocos si realmente funciona como sistema, integrando los contenidos de las diferentes materias. En la práctica esto es posible sólo cuando los actores de cada semestre y año -profesores y estudiantes- participan de forma mancomunada en el logro de sus objetivos, sin una concertación previa de las acciones pedagógicas a desarrollar que pueda responder a tales exigencias, (Silva, 2006). Desde el punto de vista de su diseño, el semestre académico se formula en términos de objetivos. Para lograr que cada uno tenga precisado con claridad su rumbo esencial, estos objetivos deben ser pocos e integradores. Deben ser el resultado de la integración de todas o de algunas de las asignaturas que se imparten. En eso radica la nueva cualidad de este subsistema. Para ello, los 
estudiantes se incorporan a proyectos o programas de la propia unidad académica o de instituciones u organizaciones en las que se desempeñan profesionales de la disciplina en posiciones laborales específicas (incluida la de investigación), refieren Ferrero y De Andrea, (2008).

La integración horizontal alude a la interconexión entre los contenidos de diversas ciencias en un momento dado, a la mezcla de sus campos de actuación, o dicho de otra forma, a la acción de mezclar de manera inteligente elementos de una asignatura con los de otra en un momento dado. Así Alvarez (1998), plantea que se realiza incluso en asignaturas con objetos distintos, mediante elementos comunes en ellas y Rosental y Ludin (1981), exponen que expresa la conexión e interdependencia de los fenómenos. Este tipo de integración es el que asume la materia designada como cátedra integradora, que se establece mediante la presentación al alumno del objeto de estudio con las características esenciales de los sistemas de conocimientos científicos y se le hace consciente que debe utilizar los conocimientos de otra ciencia para su explicación, debido a la diversidad de objetos y a los vínculos y relaciones de interdependencia entre ellos. La integración horizontal se vincula con la interconexión de los contenidos de varias ciencias en un momento dado del proceso formativo. No obstante, señalan Viera, de las Nieves y Tabares (2016), que el verdadero problema consiste en preguntarse si los profesores que imparten las diferentes asignaturas en un mismo semestre, con los mismos estudiantes, analizan colectivamente sus acciones para lograr propósitos comunes, de mayor alcance que los de cada una de las materias aisladamente.

La responsabilidad de la concepción y ejecución de la asignatura o cátedra integradora en la docencia universitaria corresponde por sus funciones a la Junta de Año que es la encargada de llevar a cabo el trabajo metodológico. Su estructura la integran los factores claves para el trabajo integrado pues agrupa a: los profesores que desarrollan las materias, al tutor y a los estudiantes. La coordinación de todos estos factores junto al apoyo de las autoridades de la carrera en cuestión y de la institución de educación superior en que estén insertadas es un proceso que no se logra rápidamente, a tal punto se tienen las experiencias de Cuba que muestran un tránsito que puede tomarse como referencia de ello, según indican Hernández, et.al., (2016):

- Etapa I. Autogestión del colectivo (junta) de año (o semestre) académico (1962-1975) En la práctica no funcionaban como colectivo y la gestión del jefe se limitaba a convocar a las juntas mensuales para analizar los resultados de las evaluaciones, los horarios de clases, su cumplimiento, la asistencia y puntualidad de los alumnos a las clases y la planificación. No obstante a estas dificultades, se propicia el trabajo educativo a través de las juntas, aunque no estuviera declarado explícitamente qué hacer y cómo hacerlo.

- Etapa II. De gestión guiada (1976-1989)

En general, en este período se mantienen las juntas de año, van emergiendo como un órgano para valorar el rendimiento académico de los estudiantes, su actividad se concentra en promover, verificar y evaluar los aspectos instructivos del proceso. Sin embargo, en esta labor se apuntaba 
al trabajo más bien individualizado de los profesores por asignaturas, que se constituían en colectivo cuando eran dos o más docentes que impartían un mismo sílabo. Aún no existía un trabajo de colectivo para la necesaria concreción de acciones que potenciaran el proceso educativo en el semestre, donde se trabajara de forma integrada para formar conocimientos, habilidades, motivos, valores en el grupo estudiantil y por consiguiente, no se lograban estrategias educativas coherentes en la dirección pedagógica porque las diferentes asignaturas incidían con enfoques más bien particulares en el proceso educativo.

- Etapa III. De gestión institucional e institucionalización remunerada de la gestión (1990-actualidad).

Los representantes de las asignaturas, con la responsabilidad de coordinar el conjunto de actividades docentes: académicas, laborales e investigativas, en correspondencia con los objetivos y atendiendo la labor instructiva y la educativa para lograr el objetivo de la formación integral de los profesionales.

Ellos eran dirigidos por un profesor guía (tutor) en el caso del grupo que eran designados por su experiencia y prestigio, gozaban de autoridad política y académica y asumían las funciones de orientación, coordinación y control asignadas, constituyéndose en la principal autoridad académica en el semestre. La tutoría se presenta en el momento actual como un elemento clave de calidad y no olvidemos que el aseguramiento de la calidad es otra de las prioridades en los rediseños curriculares según indica García et. al., (2004). Se subordina directamente al decano de la facultad-carrera.

Viera, de las Nieves y Tabares (2016), insisten en que la junta de año (semestre) es la instancia donde mayor integración académica debe existir teniendo como base el objetivo del mismo, así como la acción de buscar los procesos de descarga de contenidos entre las asignaturas.

Se ha de partir del papel rector que en el sistema de conocimientos este ocupa en la formación del profesional, tanto horizontal como transversal, de tal forma que constituya la célula básica del trabajo didáctico metodológico y donde se deben poner en práctica actividades que garanticen la integración de los contenidos para la evaluación, esto es posible a través de diversas formas del trabajo metodológico.

\section{Acciones para la integración de los contenidos en la junta de año:}

a. Determinar los objetivos del semestre académico

b. Presentación de los sílabos objeto de enseñanza para el periodo lectivo y establecer correlaciones

c. Balance del sistema de conocimientos y el papel rector de las asignaturas, eliminado la repetición o sobrecarga de estos para el estudiante

d. Determinación de contenidos a integrar

e. Identificación de las formas en que las asignaturas darán salida al objetivo del semestre 
académico y las habilidades necesarias para lograrlo.

f. Determinación de las formas de trabajo conjunto en actividades docentes a desarrollar entre asignaturas del semestre en sus múltiples formas (planificación del sistema de tareas docentes integradoras)

g. Balance del sistema de evaluación, sus formas y cuándo se desarrollará la misma priorizando las posibilidades de integración

h. Análisis de las formas de evaluación final y posibilidades de integración en exámenes finales de las asignaturas del semestre. Plantean acertadamente Pérez, Mesa y García (2013), que la evaluación del aprendizaje puede incluir aspectos teóricos y prácticos vinculados a ejercicios integradores, de modo que puedan demostrar al ser evaluados el desempeño de varias de las asignaturas que se imparten en un determinado periodo lectivo.

\section{Acciones para la integración de los contenidos en la cátedra o asignatura integradora:}

a. Identificación de los problemas profesionales de base en que pueden ser aplicados los contenidos de la asignatura para las esferas de actuación del profesional

b. Establecer la concepción metodológica de la tarea de aprendizaje o científica a resolver por los estudiantes para la integración

c. Establecer la multidisciplinariedad y las tareas a desarrollar

d. Determinación de los principales contenidos que se articulan en la integración horizontal del semestre según la malla curricular

e. Distribución de los contenidos a evaluar en las varias formas de evaluación durante el periodo lectivo:

- evaluación frecuente

- evaluaciones parciales

- estudio independiente

- evaluación final

- examen integrador

f. Establecer estrategias de aprendizaje productivo concretados en las formas de evaluación del mismo

El diseño didáctico de las actividades propuestas debe ser elaborado sobre la base del análisis del programa de las asignaturas, que exige la relación de la teoría con la práctica para alcanzar una cultura científica encaminada a resolver problemas relacionados con la vida económica, política y social del país; de ahí que se contribuya al desarrollo de habilidades en la práctica pedagógica.

La tarea docente, refieren Pérez, Mesa y García (2013), resulta una alternativa factible de utilizar para la integración, para lo que debe solicitarse al estudiante la solución de problemas profesionales de base donde sea preciso definir qué aporta cada asignatura a su solución. La realización de clases prácticas integradas en la unidad básica y la planificación de seminarios integradores que demanden el concurso de varias asignaturas, constituyen ejemplos de su aplicación en este ciclo. 
Para Viera, de las Nieves y Tabares (2016), los elementos a considerar en la elaboración de cada tarea docente integradora son:

- los resultados del diagnóstico de los alumnos

- la dosificación y el contenido de la asignatura rectora

- que el tema, por su naturaleza, se preste para la realización de un trabajo integrador

- la solución de problemas que se identifican en objetos complejos del proceso pedagógico

- los métodos, procedimientos y técnicas a utilizar

- el trabajo con las fuentes bibliográficas: consulta y referencias bibliográficas, realización de fichas, trabajo con software, etc

\section{Etapas fundamentales para la organización de la tarea integradora}

- Etapa inicial o de orientación: Se precisa de guías, textos y otros materiales que expliquen los procedimientos a emplear, los contenidos necesarios a interrelacionar, así como la preparación motivacional por parte de los docentes con objetivos definidos y las etapas de control sistemático de las actividades a ejecutar

- Etapa de ejecución o desarrollo: Se desarrollarán las acciones planeadas para alcanzar las metas propuestas, incluyendo los recursos humanos y materiales necesarios

- Etapa de final o de resultados: Se evaluará el desarrollo y ejecución de la tarea docente integradora diseñada, se valoran los resultados obtenidos y se reorienta en caso que fuera necesario.

Principales elementos a considerar en la evaluación de la tarea docente integradora: (pérez, mesa y garcía, 2013)

- Integración de conocimientos.

- Desempeño del estudiante

- Problematización de la realidad con elementos de la vida cotidiana

- Aplicación a la carrera

- Independencia

- Creatividad

- Empleo adecuado de las bibliografías y materiales de consulta

- Repercusión en su crecimiento personal y profesional

El profesor tiene que mostrar los aspectos esenciales que garanticen la realización de las actividades: actualizar los conocimientos que posee sobre el contenido de la actividad, establecer el vínculo necesario entre estos y los que adquirirán y sistematizarán durante la realización de las acciones que exigen los objetivos de la enseñanza, conservando la lógica de los conocimientos en la ejecución de la acción. Esto permite el despliegue consciente de las acciones y sus operaciones.

Es evidente que el rol del docente se complejiza y exige de mayor dedicación y preparación, así lo reconoce también Salinas (2000), quien plantea que ello conlleva nuevas responsabilidades y 
modos de hacer, ahora, el profesor universitario "no sólo debe estar al día de los descubrimientos en su campo de estudio, debe atender al mismo tiempo a las posibles innovaciones en los procesos de enseñanza-aprendizaje y en las posibilidades de las tecnologías de la información y la comunicación. Debe prepararse para un nuevo rol de profesor como guía y facilitador de recursos y que orienten a alumnos activos que participen en su propio proceso de aprendizaje". Esto hace que: "además detener la responsabilidad del contenido, el profesor haya de participar en el proceso de diseño y elaboración de los materiales de aprendizaje en los procesos de distribución de los mismos y en los procesos interactivos de intercambio de información, opiniones y experiencias o en las tutorías, así como en la actualización y mejora de los materiales..."

La lógica de la acción queda, según Castro (2016), organizada en una estructura didáctica, que para García (1993), lo constituye la guía didáctica como “...el documento que orienta el estudio, acercando a los procesos cognitivos del alumno el material didáctico, con el fin de que pueda trabajarlos de manera autónoma”, la guía didáctica adquiere una importancia tal, que al respecto señala: “...en realidad una guía didáctica bien elaborada y al servicio del estudiante, debería ser un elemento motivador de primer orden para despertar el interés por la materia o materias correspondientes. Debe ser instrumento idóneo para guiar y facilitar el aprendizaje, ayuda a comprender y a aplicar los diferentes conocimientos, así como para integrar todos los medios y recursos que se presentan al estudiante como apoyo para su aprendizaje...”.

Otra definición la aporta Martínez(2000), al afirmar que: “... constituye un instrumento fundamental para la organización del trabajo del alumno y su objetivo es ofrecer todas las orientaciones necesarias que le permitan integrar los elementos didácticos para el estudio de las asignaturas".

Las guías didácticas constituyen un recurso esencial del cual no se debe prescindir en los procesos de aprendizaje y de integración. Sobre este medio, cuyo objetivo es enfatizar en su uso por la significación que adquiere actualmente para optimizar las labores del profesor y del estudiante también la apoyan García y de la Cruz (2014). Generalmente estas guías se asocian a la educación a distancia o la modalidad semipresencial, lo cual constituye un error, ya que una educación presencial que abogue por la autonomía del aprendizaje, requiere también necesariamente que los profesores elaboren guías que les permitan no solo orientar, sino también contribuir a la organización del trabajo del estudiante y el suyo propio.

\section{Exigencias didácticas para la integración curricular}

No es suficiente solo tener un buen diseño metodológico de la carrera, lo complejo es organizar y garantizar que funcionen sistemáticamente, sin insuficiencias ni contradicciones antagónicas, todas las estructuras académicas en su aplicación. La cátedra integradora debe convocar y coordinar las acciones con: la carrera, la junta de año y las asignaturas participantes. 
Se comparte el criterio de Flor y Garritz (2006), que es una propuesta metodológica que requiere mucho tiempo del profesor para la preparación de sus clases, pues debe innovar sus recursos pedagógicos y proponer nuevas didácticas que posibiliten el desarrollo de las competencias referidas en el perfil profesional. La participación del docente en este proceso consiste en favorecer el proceso de reflexión, la adquisición y profundización de fundamentos teóricos que serán la base de las soluciones y reflexiones.

Es un sistema que demanda una intensa y rigurosa planificación de los conocimientos técnicos y científicos que serán impartidos en cada una de las materias y de cómo estos se pondrán en práctica para que el estudiante se apropie del conocimiento.

Otro problema actual consiste en lograr que los profesores que imparten las diferentes asignaturas en un mismo semestre, con los mismos estudiantes, analicen colectivamente sus acciones para lograr propósitos comunes, de mayor alcance que los de cada una de las materias aisladamente.

La función de la cátedra integradora sólo es posible si el profesor conoce de qué forma se ha establecido la estrategia formativa de las asignaturas precedentes y cómo se realizará el abordaje de las posteriores, lo cual implica que además de estudiar los documentos del diseño, el perfil declarado y las unidades curriculares, deben existir momentos de análisis tanto en el sentido horizontal como vertical del proceso formativo.

La formación continua de los docentes, la autopreparación y la autosuperación, que se origina desde el trabajo metodológico, diseñado a partir de la concepción de integración, tiene su base en la necesidad actual de que los docentes conozcan y apliquen en el desarrollo de la docencia y la investigación, las técnicas más avanzadas de la informática y los presupuestos teóricos de las ciencias pedagógicas, psicológicas, filosóficas y sociológicas, en correspondencia con una alta preparación científica en su ciencia y una adecuada actualización de sus conocimientos, apuntan Gutiérrez y Domínguez (2003).

\section{CONCLUSIONES}

La integración, que como función, se le adjudica en el semestre académico a la cátedra integradora en los rediseños curriculares, satisface a la necesidad de lograr un enfoque sistémico del contenido de la enseñanza y formar un futuro profesional que desde la formación básica esté en condiciones de resolver los problemas más frecuentes y generales que se presenten en las disímiles esferas de su profesión. Por su subordinación necesita del apoyo de la carrera y como eslabón base de la junta del semestre o año, ya que es allí donde se concretan las metas de la organización que los niveles superiores no podrán sustituir, la tendencia hoy es descentralizar al máximo posible las decisiones sobre los procesos que se desarrollan y que los profesores con más experiencia y condiciones ocupen las responsabilidades para conducir metodológicamente en la base reformando estructuras, métodos de trabajo y recursos de aprendizaje que contribuyan a la interconexión horizontal de 
saberes y que además exige de la superación continua de todos los docentes. Las condiciones y necesidades del estudiante universitario actual han cambiado y ahora se demanda un mayor asesoramiento no sólo en el ámbito académico, sino también en cuestiones sociales, personales y profesionales.

\section{REFERENCIAS BIBLIOGRÁFICAS}

Álvarez de Zayas, C. M. (1998). Pedagogía como ciencia. Habana - Cuba. Editora Universidad de La Habana.

Aldana, G. y Joya, N. (2008). Actitudes hacia la investigación científica de los docentes que orientan la asignatura de metodología de investigación. Educación y educadores. Universidad de La Sabana. vol.11 no.2 ISSN 0123-1294 pp 61-68

Avilés Dinarte, G. (2011). La metodología indagatoria: una mirada hacia el aprendizaje significativo desde "Charpack y Vigotsky". Revista de las Sedes Regionales de la Universidad de Costa Rica. Vol. XII, N²3. ISSN: 2215-2458 133.144pp

Castro Ramos, Y. (2016). "El desarrollo de habilidades prácticas en la formación inicial del profesor de biología”, Revista Atlante: Cuadernos de Educación y Desarrollo ISSN: 1989-4155

Cuevas De la Garza, María S. (2009). La participación de los profesores universitarios en una reforma curricular a partir de su conocimiento profesional práctico. T e s i s para obtener el grado de Doctora en Educación. Instituto Tecnológico y de Estudios Superiores de Occidente. Departamento de Psicología, Educación y Salud. 309 pp

Cuza Sánchez, J.C. y Guibo Silva, A. (2015). La integración universidad - escuela politécnicaentidad laboral, para la formación del profesional de la Educación Técnica y Profesional. EduSol, ISSN: 1729-8091. Vol. 15, No. 51. 31-40 pp

Davidov, V. (1987). "Análisis de los principios didácticos de la escuela tradicional y posibles cambios de enseñanza en el futuro próximo”, en Psicología evolutiva y pedagógica en la URSS. Antología. Moscú: Editorial Progreso.

Ferrero, A., y De Andrea, N. (2008). Relevancia de las prácticas pre-profesionales en la formación universitaria. El caso de la psicología. En XV Jornadas de Investigación y Cuarto Encuentro de Investigadores en Psicología del Mercosur. Facultad de Psicología-Universidad de Buenos Aires.

Flor Reyes, C. y Garritz, A. (2006). Conocimiento pedagógico del concepto de "Reacción química" en profesores universitarios mexicanos. Revista Mexicana de Investigación Educativa., VOL. 11, NÚM. 31, PP. 1175-1205

García Aretio, L. (1993). Componentes básicos de un curso a distancia. En Revista Iberoamericana de Educación Superior a Distancia, Vol. 5, n 3, pp. 61-80.

García Hernández, I., de la Cruz Blanco, G.M. (2014). Las guías didácticas: recursos necesarios para el aprendizaje autónomo. Revista EDUMECENTRO vol.6 no.3

García Nieto, N., Asensio Muñoz, I., Carballo Santaolalla, R., García García, M. y Guardia González, S. (2004). Trabajo subvencionado por el MECD en el Programa de Estudios y 
Análisis de la Dirección General de Universidades, como acción destinada a la mejora de la calidad de la Enseñanza Superior y de Actividades del Profesorado. Secretaría de Estado de Educación y Universidades. Madrid.

Gutiérrez, T. B., y Domínguez, T. D. (2003). Un modelo de trabajo metodológico en el año académico y su contribución a la formación continua del profesorado. Pedagogía Universitaria, 8(5).

Hernández, B. T., Recasens, J. R., González, O. L. P., y Ramírez, M. G. (2016). Gestión de los colectivos de años académicos en la educación superior cubana: tendencias históricas. Academia y Virtualidad, 9(1), 41-51.

Martínez Mediano C. (2000). Elaboración de materiales didácticos escritos para la educación a distancia. Revista Enseñanza e Investigación en Psicología. 5(33):33-50.

Perafán, G. (2004). La epistemología del profesor sobre su propio conocimiento profesional. Bogotá: Universidad Pedagógica Nacional

Pérez Benítez I.M., Mesa Herrera, G. y García Naranjo, M.A. (2013). La tarea integradora y su evaluación en la docencia universitaria. Disponible en: http:/www.cubaeduca.cu/medias/ evaluador/Ines-Maria.pdf. Consultado: 27/09/2016 a 10:45

Rosental, M. y Ludin, P. (1981). Diccionario Filosófico. La Habana. Editorial Pueblo y Educación. $536 \mathrm{pp}$.

Salinas, J. (2000). El rol del profesorado en el mundo digita". En Del Carmen, L. (ed.). Simposio sobre la formación inicial de los profesionales de la educación. Universitat de Girona.

Sierra Figueredo, S., Fernández Sacasas, J. A., Miralles Aguilera, E., Pernas Gómez, M., y Diego Cobelo, J. M. (2009). Las estrategias curriculares en la Educación Superior: su proyección en la Educación Médica Superior de pregrado y posgrado. Educación Médica Superior, vol 5,no 3 , p. $212-224$,

Silva, P. H. (2000). EL modelo curricular de la Educación Superior Cubana. Pedagogía Universitaria. Vol. 5 No. 3. 1-4pp

Silva, P. H. (2006). El proceso de formación en la universidad cubana. Pedagogía Universitaria, 11(3).

Valdés Carrillo A. G., Valdés Carrillo, A.M., Fernández Oliva, B. (2016). Estrategia pedagógica para elevar la formación vocacional y orientación profesional de los estudiantes de Enfermería en la Facultad "Gral. Calixto García” Educación Médica Superior. vol.30 no.2

Viera Sánchez, B. E., de las Nieves Díaz-Hernández, M., y Tabares Arévalo, R. M. (2016). Guía metodológica para la integración de la evaluación en el plan de estudio de la Facultad de Cultura Física "Nancy Uranga Romagoza”. Revista Podium, 8(2), 33-44. 